\title{
Application of the discrete element method (DEM) to particle laden multi-phase flows
}

\author{
Harald Kruggel-Emden \\ Mechanical Process Engineering and Solids Processing (MVTA), \\ Technische Universität Berlin, Berlin, Germany \\ E-mail:kruggel-emden@tu-berlin.de
}

\begin{abstract}
The discrete element method (DEM) coupled with methods for computational fluid dynamics (CFD) is a very capable instrument to capture particle-laden multi-phase flows. While often the flow around individual particles is not resolved on the CFD-side particle interaction is captured in detail. More recently resolved flow simulations have gained in popularity which can be also based on the Lattice Boltzmann method (LBM). By coupling the latter with the DEM particle/fluid systems can even be studied in more detail. A brief review of both approaches is performed and possible applications are discussed.
\end{abstract}

Keywords: simulations, multi-phase flows, DEM-CFD, FVM, LBM, non-spherical particles.

\section{Introduction}

The discrete element method (DEM) has established itself as a powerful tool for modelling static or dynamic particle systems in the past 10 years. While in the beginning of the DEM dating back to Cundall and Strack in 1979 (Cundall \& Strack, 1979) particles always were considered to be of spherical shape mostly due to runtime issues, they can for some time be non-spherical (comp. e.g. (Cleary, 2008; Markauskas, Kačianauskas, Džiugys, \& Navakas, 2010; Höhner, Wirtz, Kruggel-Emden, \& Scherer, 2011) which allows simulations in a wide range of engineering disciplines to capture real world processes and phenomena related to particle systems in a very precise manner. In recent years also coupled fluid/particle flows have shifted into the focus. Even in such flows the non-sphericity of particles has become an issue as it can have an influence on the overall behavior of the particle/fluid system (comp. e.g. (Hilton, Mason, \& Cleary, 2010; Zhou, Pinson, Zou, \& Yu, 2011; Ren et al., 2012; Kruggel-Emden \& Oschmann, 2014; Oschmann, Hold, \& Kruggel-Emden, 2014).

Research in the area of particle laden multi-phase flows is advancing fast. Simulations up to now have mostly been performed by coupling the DEM with computational fluid dynamics (CFD) not resolving the flow around individual particles. Thereby the CFD usually relies on the discretization of the Navier-Stokes equations based on the Finite Volume Method (FVM); however more recently other CFD approaches have also started to be more widespread used. At the moment resolved flow simulations have gained in popularity basing on the Lattice Boltzmann method (LBM) (see e.g. (Kravets, Rosemann, Reinecke, \& Kruggel-Emden, 2019)) as they allow to dispense the use of empirical closures for momentum and possible heat as well as mass transfer between particles and the fluid phase. The two latter methods are briefly reviewed in the following and examples for their application given.

\section{Particle unresolved DEM-FVM based CFD}

In the coupled DEM-FVM based CFD method, the particle motion is modelled within a DEM-framework, while the fluid phase is represented by solving the volume averaged Navier-Stokes equations (Zhu, Zhou, Yang, \& Yu, 2007). In the DEM the translational and rotational motion is obtained by integrating Newton's and Euler's equations of each particle given by

$$
m_{i} \frac{d^{2} \vec{x}_{i}}{d t^{2}}=\vec{F}_{i}^{c}+\vec{F}_{i}^{p f}+m_{i} \vec{g}
$$




$$
\hat{I}_{i} \frac{d \vec{W}_{i}}{d t}+\vec{W}_{i} \times\left(\hat{I}_{i} \vec{W}_{i}\right)=\Lambda_{i}^{-1}\left(\vec{M}_{i}\right)=\Lambda_{i}^{-1}\left(\vec{M}_{i}^{c}+\vec{M}_{i}^{p f}\right),
$$

with the particle mass $m_{i}$, particle acceleration $d^{2} \vec{x}_{i} / d t^{2}$, contact force $\vec{F}_{i}^{c}$, particle/fluid force $\vec{F}_{i}^{p f}$, gravitational force $m_{i} \vec{g}$, angular acceleration $d \vec{W}_{i} / d t$, angular velocity $\vec{W}_{i}$, external moment $\vec{M}_{i}$ resulting out of contact $\vec{M}_{i}^{c}$ or particle/fluid forces $\vec{M}_{i}^{p f}$, the inertia tensor along the principal axis $\hat{I}_{i}$ and the rotation matrix converting a vector from the inertial into the body fixed frame $\Lambda_{i}^{-1}$ with $i$ the particle index.

A flexible approach to model complex shaped particles in the discrete element method is the representation by polyhedrons. In this method a triangular surface mesh is applied to represent the desired particle shape and a common plane algorithm is used for contact detection (Höhner et al., 2011). Contact force laws are applied similarly as used for spherical particles (Kruggel-Emden, Simsek, Rickelt, Wirtz, \& Scherer, 2007a; Kruggel-Emden, Wirtz, \& Scherer, 2007b). The normal component of the contact forces is obtained from a linear spring damper model

$$
\vec{F}^{n}=k^{n} \delta \vec{n}+\gamma^{n} \vec{v}_{r e l}^{n},
$$

where: $k^{n}$ is the spring stiffness, $\delta$ the virtual overlap, $\vec{n}$ a normal vector, $\gamma^{n}$ a damping coefficient and $\vec{v}_{\text {rel }}^{n}$ the normal velocity in the contact point. Both $k^{n}$ and $\gamma^{n}$ determine the coefficient of normal restitution between particles $e_{P P}^{n}$ as well as particles and walls $e_{P W}^{n}$. For the calculation of the tangential forces a linear spring limited by the Coulomb condition is used

$$
\vec{F}^{t}=\min \left(k^{t}\left|\vec{\xi}^{t}\right|, \mu_{C}\left|\vec{F}^{n}\right|\right) \vec{t},
$$

where: $k^{t}$ is the stiffness of a linear spring, $\mu_{C}$ is the friction coefficient, $\vec{\xi}^{t}$ is the relative tangential displacement and $\vec{t}$ is the tangential unit vector. Rolling friction can additionally be considered.

The fluid phase is described with Computational Fluid Dynamics (CFD) in an Eulerian framework. The fluid velocity is considered as a spatially averaged quantity on the cell level. The CFD-framework passes the fluid properties and the velocity of the fluid to the DEM. The equation of continuity (5) and the equation of momentum (6) are solved

$$
\begin{gathered}
\frac{\partial\left(\varepsilon_{f} \rho_{f}\right)}{\partial t}+\nabla\left(\varepsilon_{f} \rho_{f} \overrightarrow{u_{f}}\right)=0 \\
\frac{\partial\left(\varepsilon_{f} \rho_{f} \overrightarrow{u_{f}}\right)}{\partial t}+\nabla\left(\varepsilon_{f} \rho_{f} \overrightarrow{u_{f}} \overrightarrow{u_{f}}\right)=-\varepsilon_{f} \nabla \mathrm{p}+\nabla\left(\overline{\overline{\varepsilon_{f} \tau}}\right)+\varepsilon_{f} \rho_{f} \vec{g}+\vec{f}_{i n t},
\end{gathered}
$$

where $\vec{u}_{f}, \rho_{f}$ and $\mathrm{p}$ are the physical fluid velocity, density and pressure. $\vec{f}_{\text {int }}$ is the volumetric particle/fluid interaction momentum source applied in each CFD cell, $\varepsilon_{f}$ is the local fluid porosity and $\bar{\tau}$ is the fluid viscous stress tensor $\stackrel{=}{\tau}=\eta_{\mathrm{e}}\left[\left(\nabla \vec{u}_{f}\right)+\left(\nabla \vec{u}_{f}\right)^{-1}\right]$ with $\eta_{\mathrm{e}}$ the effective viscosity determined from a possible turbulent model. The particle/fluid interaction $\vec{f}_{i n t}$ is given component wise as $f_{\text {int }}=\bar{\beta}_{j}\left(u_{f, j}-\bar{v}_{j}\right)$, where $\bar{v}_{j}$ is the fluid cell averaged particle velocity, and $\bar{\beta}_{j}$ is the fluid cell averaged particle/fluid friction coefficient with $j=x, y, z$.

The particle/fluid force $\vec{F}_{i}^{p f}$ consists of all individual particle/fluid forces such as drag force $\vec{F}_{i}^{d}$ and pressure gradient force $\vec{F}_{i}^{\nabla p}$ acting on a particle $i$ written as $\vec{F}_{i}^{p f}=\vec{F}_{i}^{d}+\vec{F}_{i}^{\nabla p}$. Both can be combined to $\vec{F}_{i}^{d}+\vec{F}_{i}^{\nabla p}=\beta_{j} V_{i}\left(\vec{u}_{f}-\vec{v}_{i}\right) /\left(\varepsilon_{f}\left(1-\varepsilon_{f}\right)\right)$, where $V_{i}$ is the particle volume of particle $i$ and $j=x, y, z$. For the calculation of the drag force $\vec{F}_{i}^{d}$ and the combined drag and pressure gradient force $\vec{F}_{i}^{d}+\vec{F}_{i}^{\nabla p}$ various models are available. Very popular and widely used for spherical particles and also applicable for non-spherical particles is the approach by Di Felice (Di Felice, 1994), where the force for an isolated spherical particle is calculated and altered by the influence of surrounding particles. The respective force reads

$$
\vec{F}_{i}^{p f}=\vec{F}_{i}^{d}+\vec{F}_{i}^{\nabla p}=\frac{1}{2} \rho_{f}\left|\vec{u}_{f}-\vec{v}_{i}\right| C_{D} A_{\perp} \varepsilon_{f}^{1-\chi}\left(\vec{u}_{f}-\vec{v}_{i}\right),
$$

where $C_{D}$ is the drag coefficient, $A_{\perp}$ is the cross-sectional area perpendicular to the flow and $\chi$ a correction factor. Eq. (7) can be rewritten in terms of the particle/fluid friction coefficient as

$$
\vec{\beta}_{i}=\frac{1}{2} \rho_{f} C_{D} A_{\perp} \varepsilon_{f}\left|\vec{u}_{f}-\vec{v}_{i}\right|\left(1-\varepsilon_{f}\right) \frac{1}{V_{i}} \varepsilon_{f}^{(1-\chi)} .
$$


$\chi$ is calculated as a function of the Reynolds-number $R e=\varepsilon_{f} \rho_{f} d_{e}\left|\vec{u}_{f}-\vec{v}_{i}\right| / \eta_{\mathrm{f}}$ as

$$
\chi=3.7-0.65 \exp \left(-(1.5-\log (R e))^{2} / 2\right)
$$

with $d_{e}$ the diameter of a volume equivalent spherical particle and $\eta_{\mathrm{f}}$ the fluid viscosity. The drag coefficient $C_{D}$ of a single particle (spherical or non-spherical) can be derived from correlations such as Hölzer und Sommerfeld (Hölzer and Sommerfeld, 2008) which can be written as

$$
C_{D}=\frac{8}{\operatorname{Re}} \frac{1}{\sqrt{\phi_{\perp}}}+\frac{16}{\operatorname{Re}} \frac{1}{\sqrt{\phi}}+\frac{3}{\sqrt{\operatorname{Re}}} \frac{1}{\phi^{3 / 4}}+0.42 \times 10^{0.4(-\log (\phi))^{0.2}} \frac{1}{\phi_{\perp}}
$$

where $\phi_{\perp}$ is the crosswise sphericity which is the ratio between the cross-sectional area of the volume equivalent sphere and the projected cross-sectional area of the considered particle perpendicular to the flow.

The porosity $\varepsilon_{f}$ can be calculated based on a subgrid cell approach (Oschmann et al., 2014). A finer resolved grid is examined and it is checked if a particular subgrid cell center lies within a part of a particle. If this is the case the respective subcell is considered as fully filled with solid. Based on the obtained ratio the local fluid porosity is calculated (Oschmann et al., 2014)..

\section{Particle resolved DEM-LBM based CFD}

In order to perform particle resolved DEM-CFD simulations, the Lattice Boltzmann method (LBM) as CFD approach has gained increasing popularity, due to its simplicity as well as its good scalability (Succi, 2008, 2015; He, Liu, Li, \& Tao, 2019). As a direct numerical method (DNS), the LBM is able to resolve the flow around individual particles providing a detailed understanding of the interaction between the particle and fluid phase. For hydrodynamic problems, the Lattice Boltzmann method recovers the solution of the incompressible Navier-Stokes equation. Lattice Boltzmann models require the definition of a collision operator describing the interaction of distribution functions within the fluid domain.

The collision of density distribution functions $f_{i}$ in a node located at $r=(x, y, z)^{\mathrm{T}}$ in the fluid domain and the subsequent streaming in one time step $\Delta t$ along the lattice velocity $e_{i}$ to the neighboring node at $r+e_{i} \Delta t$ can be written as

$$
f_{i}\left(r+e_{i} \Delta t, t+\Delta t\right)=f_{i}(x, t)-\Omega_{i}(f), \quad i=1,2, \ldots n,
$$

where the collision is described by the collision operator $\Omega_{i}(f)$ which is described later. The D3Q19 lattice $(n=19)$ which is often adopted includes the discrete velocities

$$
e_{i}=\left\{\begin{array}{cl}
(0,0,0), & i=1, \\
( \pm 1,0,0) c,(0, \pm 1,0) c,(0,0, \pm 1) c, & i=2-7 \\
(0, \pm 1, \pm 1) c,( \pm 1,0, \pm 1) c,( \pm 1, \pm 1,0) c, & i=8-19
\end{array}\right.
$$

where $c=\Delta x / \Delta t$ is the lattice speed which is often set to 1 for convenience.

Based on the distribution functions the local density $\rho$, the momentum $j$ and the velocity $u=(u, v, w)^{\mathrm{T}}$ can be obtained by

$$
\rho=\sum_{i=1}^{n} f_{i} \quad \text { and } \quad j=\rho_{0} u=\sum_{i=1}^{n} f_{i} e_{i} .
$$

In the MRT model by d'Humières (d'Humières, 2002), distribution functions are transformed into the moment space $m$ via

$$
m(r, t)=M f(r, t)
$$

with the transformation matrix $M$ defined as 


$$
M=\left[\begin{array}{ccccccccccccccccccc}
1 & 1 & 1 & 1 & 1 & 1 & 1 & 1 & 1 & 1 & 1 & 1 & 1 & 1 & 1 & 1 & 1 & 1 & 1 \\
0 & 1 & -1 & 0 & 0 & 0 & 0 & 0 & 0 & 0 & 0 & 1 & -1 & -1 & 1 & 1 & -1 & 1 & -1 \\
0 & 0 & 0 & 1 & -1 & 0 & 0 & 1 & -1 & 1 & -1 & 0 & 0 & 0 & 0 & 1 & -1 & -1 & 1 \\
0 & 0 & 0 & 0 & 0 & 1 & -1 & 1 & -1 & -1 & 1 & 1 & -1 & 1 & -1 & 0 & 0 & 0 & 0 \\
0 & 2 & 2 & -1 & -1 & -1 & -1 & -2 & -2 & -2 & -2 & 1 & 1 & 1 & 1 & 1 & 1 & 1 & 1 \\
0 & -4 & -4 & 2 & 2 & 2 & 2 & -2 & -2 & -2 & -2 & 1 & 1 & 1 & 1 & 1 & 1 & 1 & 1 \\
0 & 0 & 0 & 1 & 1 & -1 & -1 & 0 & 0 & 0 & 0 & -1 & -1 & -1 & -1 & 1 & 1 & 1 & 1 \\
0 & 0 & 0 & 0 & 0 & 0 & 0 & 1 & 1 & -1 & -1 & 0 & 0 & 0 & 0 & 0 & 0 & 0 & 0 \\
0 & 0 & 0 & 0 & 0 & 0 & 0 & 0 & 0 & 0 & 0 & 1 & 1 & -1 & -1 & 0 & 0 & 0 & 0 \\
0 & 0 & 0 & 0 & 0 & 0 & 0 & 0 & 0 & 0 & 0 & 0 & 0 & 0 & 0 & 1 & 1 & -1 & -1 \\
-30 & -11 & -11 & -11 & -11 & -11 & -11 & 8 & 8 & 8 & 8 & 8 & 8 & 8 & 8 & 8 & 8 & 8 & 8 \\
12 & -4 & -4 & -4 & -4 & -4 & -4 & 1 & 1 & 1 & 1 & 1 & 1 & 1 & 1 & 1 & 1 & 1 & 1 \\
0 & -4 & 4 & 0 & 0 & 0 & 0 & 0 & 0 & 0 & 0 & 1 & -1 & -1 & 1 & 1 & -1 & 1 & -1 \\
0 & 0 & 0 & -4 & 4 & 0 & 0 & 1 & -1 & 1 & -1 & 0 & 0 & 0 & 0 & 1 & -1 & -1 & 1 \\
0 & 0 & 0 & 0 & 0 & -4 & 4 & 1 & -1 & -1 & 1 & 1 & -1 & 1 & -1 & 0 & 0 & 0 & 0 \\
0 & 0 & 0 & -2 & -2 & 2 & 2 & 0 & 0 & 0 & 0 & -1 & -1 & -1 & -1 & 1 & 1 & 1 & 1 \\
0 & 0 & 0 & 0 & 0 & 0 & 0 & 0 & 0 & 0 & 0 & -1 & 1 & 1 & -1 & 1 & -1 & 1 & -1 \\
0 & 0 & 0 & 0 & 0 & 0 & 0 & 1 & -1 & 1 & -1 & 0 & 0 & 0 & 0 & -1 & 1 & 1 & -1 \\
0 & 0 & 0 & 0 & 0 & 0 & 0 & -1 & 1 & 1 & -1 & 1 & -1 & 1 & -1 & 0 & 0 & 0 & 0
\end{array}\right] .
$$

The collision operator for the MRT model can be written as

$$
\Omega(f)=M^{-1} S\left[m-m^{e q}\right] .
$$

The macroscopic moments are

$$
m=\left(\rho, j_{x}, j_{y}, j_{z}, 3 p_{x x}, 3 \pi_{\mathrm{xx}}, p_{w w}, p_{y z}, p_{x z}, p_{x y}, e, \varepsilon, q_{x}, q_{y}, q_{z}, \pi_{w w}, m_{x}, m_{y}, m_{z}\right)^{T}
$$

where the first four hydrodynamic moments for density and momentum are conserved and the other variables are nonconserved kinetic moments. The equilibrium moments only depend on the conserved moments and can be computed as

$$
m^{e q}=\left(\rho, j_{x}, j_{y}, j_{z}, \frac{1}{\rho_{0}}\left(2 j_{x}^{2}-j_{y}^{2}-j_{z}^{2}\right), 0, \frac{1}{\rho_{0}}\left(j_{y}^{2}-j_{z}^{2}\right), \frac{j_{y} j_{z}}{\rho_{0}}, \frac{j_{x} j_{z}}{\rho_{0}}, \frac{j_{x} j_{y}}{\rho_{0}},-11 \rho+\frac{19}{\rho_{0}} j \cdot j,-\frac{475}{63 \rho_{0}} j \cdot j,-\frac{2}{3} j_{x},-\frac{2}{3} j_{y},-\frac{2}{3} j_{z}, 0,0,0,0\right)^{T},
$$

where the system's mean density $\rho_{0}$ is often used to reduce compressibility effects (d'Humières, 2002). The advantage of the MRT model is that the individual moments can be relaxed towards their equilibrium with different rates $s_{i}$ which are defined in the matrix

$$
S=\operatorname{diag}\left(0,0,0,0, s_{v}, s_{\pi}, s_{v}, s_{v}, s_{v}, s_{v}, s_{e}, s_{\varepsilon}, s_{q}, s_{q}, s_{q}, s_{\pi}, s_{m}, s_{m}, s_{m}\right) .
$$

The relaxation rate $s_{v}$ is connected to the model's kinematic viscosity

$$
v=c_{s}^{2}\left(\tau_{v}-\frac{1}{2}\right) \Delta t=c_{s}^{2}\left(\frac{1}{s_{v}}-\frac{1}{2}\right) \Delta t
$$

where $\tau_{v}$ denotes the relaxation time. The other relaxation rates are free parameters which can be used to optimize the performance of the MRT model. Often $s_{\pi}=s_{\varepsilon}=1.4, s_{e}=1.19$ and $s_{m}=1.98$ to improve the stability as suggested by d'Humières (d'Humières, 2002). Furthermore, following Verhaeghe et al. (Verhaeghe, Luo, \& Blanpain, 2009) $s_{q}=8\left(2-s_{v}\right) /\left(8-s_{v}\right)$ in order to remove the viscosity-dependence in parts.

Besides inflow and outflow boundary conditions a popular way to satisfy the no-slip boundary condition on particle surfaces or walls is the application of bounce-back schemes, the simplest of which is the halfway bounce-back

$$
f_{\bar{i}}\left(r_{\mathrm{f}}, t+\Delta t\right)=\tilde{f}_{i}\left(x_{\mathrm{f}}, t\right)+2 \omega_{i} \rho_{0} \frac{u_{\mathrm{b}} \cdot e_{\bar{i}}}{c_{\mathrm{s}}^{2}}
$$

where the post-collision distribution $\tilde{f}_{i}\left(x_{\mathrm{f}}, t\right)$ is reflected at the boundary during the streaming to get the missing pre-collision distribution $f_{\bar{i}}\left(r_{\mathrm{f}}, t+\Delta t\right)$. Due to the boundary's motion with velocity $u_{\mathrm{b}}$, a portion of mass is covered or uncovered by the particle which is added to the reflected distribution. The local boundary velocity $u_{\mathrm{b}}=u_{\mathrm{p}}+\omega_{\mathrm{p}} \times r_{\mathrm{b}}$ 
at the boundary position $r_{\mathrm{b}}$ takes into account the particle's translational velocity $u_{\mathrm{p}}$ as well as the rotational velocity $\omega_{\mathrm{p}}$.

For the coupling of the LBM with the DEM it is essential to evaluate the particle-fluid forces in particulate flows, which can be efficiently done in LB simulations through the momentum exchange method (MEM). The main idea of this method is to sum up the momentum change over all boundary links of a particle. In the case of moving particles, the formulation of the MEM has a great impact on accuracy and stability (Peng, Teng, Hwang, Guo, \& Wang, 2016; Tao, Hu, \& Guo, 2016). Often the force evaluation for the particle-fluid force $F_{\mathrm{pf}}$ by Wen et al. (Wen, Zhang, Tu, Wang, \& Fang, 2014) is adopted, where the boundary velocity is explicitly taken into account to obtain stable results:

$$
\vec{F}_{\mathrm{pf}}=\frac{\Delta x^{3}}{\Delta t} \sum_{r_{\mathrm{f}} i_{\mathrm{bl}}}\left[\tilde{f}_{i}\left(r_{\mathrm{f}}, t\right)\left(c_{i}-u_{\mathrm{b}}\right)-f_{\bar{i}}\left(r_{\mathrm{f}}, t+\Delta t\right)\left(c_{\bar{i}}-u_{\mathrm{b}}\right)\right] .
$$

The moment can be computed along with the force as follows:

$$
\vec{M}_{\mathrm{pf}}=\frac{\Delta x^{3}}{\Delta t} \sum_{r_{\mathrm{f}} i_{\mathrm{bl}}}\left(r_{\mathrm{b}}-r_{\mathrm{p}}\right)\left[\tilde{f}_{i}\left(r_{\mathrm{f}}, t\right)\left(c_{i}-u_{\mathrm{b}}\right)-f_{\bar{i}}\left(r_{\mathrm{f}}, t+\Delta t\right)\left(c_{\bar{i}}-u_{\mathrm{b}}\right)\right],
$$

where $r_{\mathrm{p}}$ is the position of the particle centre. Both $\vec{F}_{\mathrm{pf}}$ and $\vec{M}_{\mathrm{pf}}$ can be introduced into Eqs. (1) and (2) resembling the particle resolved DEM-LBM based CFD.

\section{Possible applications of particle unresolved DEM-FVM and resolved DEM-LBM}

Particle unresolved DEM-FVM approaches have been applied to a variety of applications so far. As they are computationally less demanding than particle resolved approaches basing on the DEM-LBM they can be applied to the modelling of whole apparatuses or technical processes. Some examples for applications are given in Figure 1.

a)
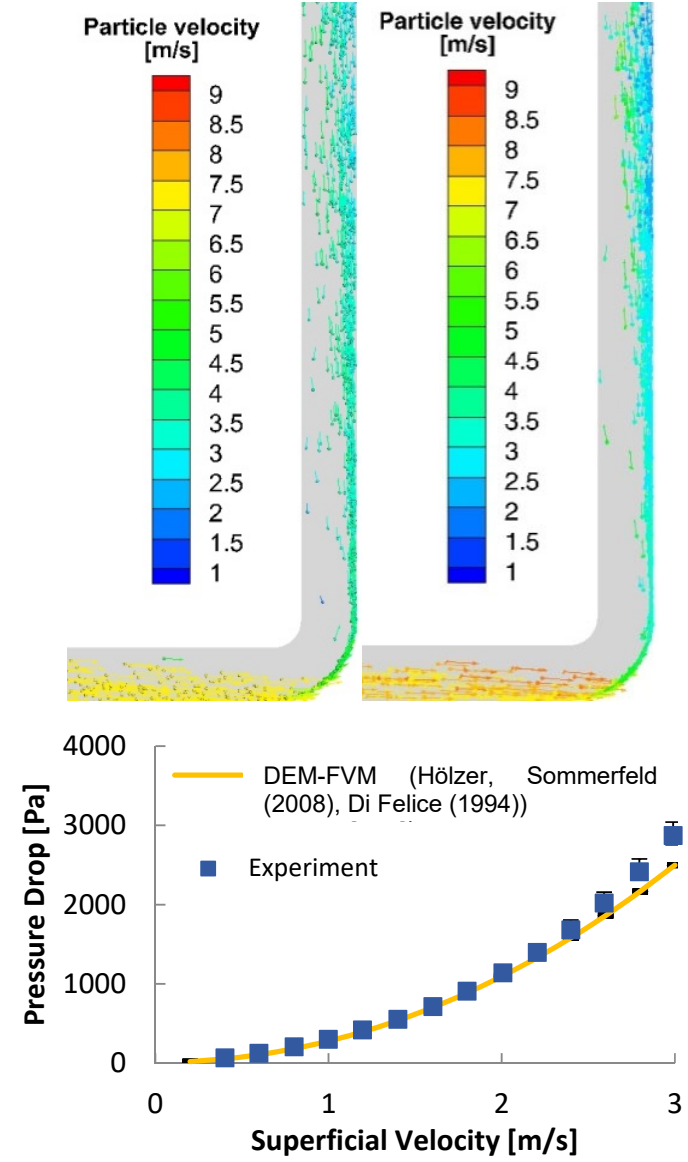

b)
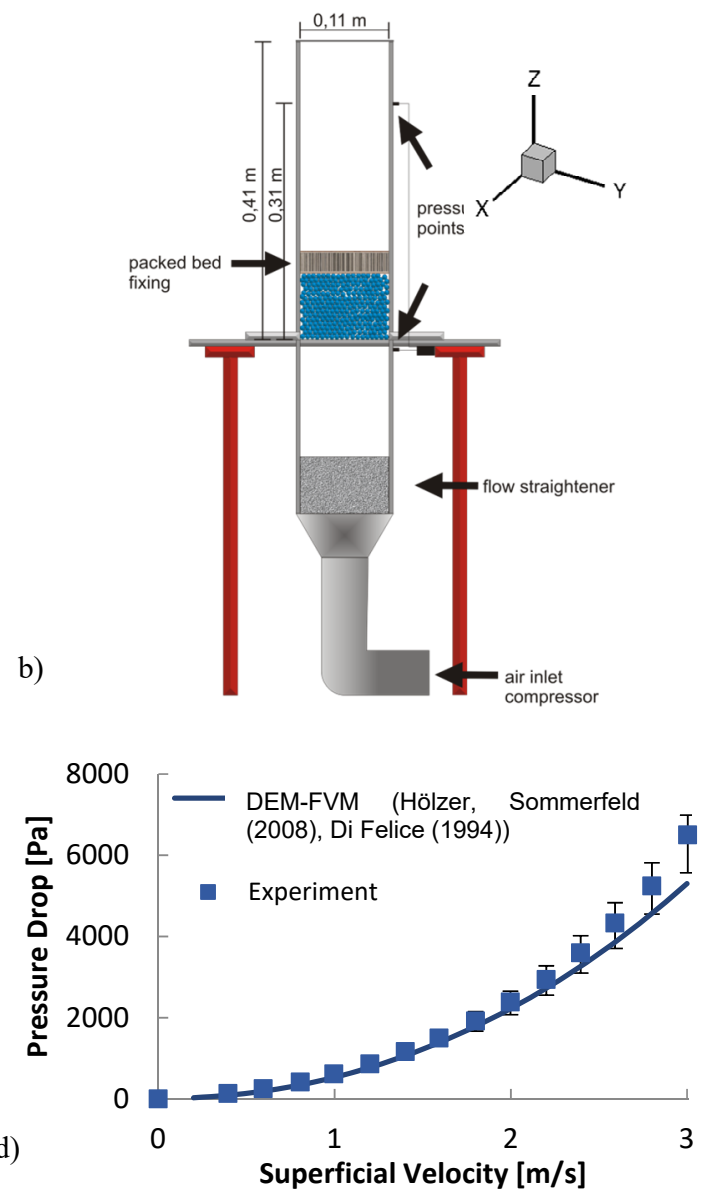

Figure 1. (a) Particle velocities of spherical particles (left) and plate shaped particles (right) in a bend being part of a pneumatic conveying system; (b) experimental setup to perform pressure drop investigations in a packed bed; (c) pressure drop of $\mathrm{d}=7 \mathrm{~mm}$ spherical particles and (d) pressure drop of $6 \mathrm{~mm} \times 6 \mathrm{~mm}$ cylinders 
One application of unresolved DEM-FVM are pneumatic conveying systems which comprise of vertical and horizontal sections which are often interconnected by bends. In such systems (comp. Figure 1a) a variety of bulk solids is transported in industrial applications. The particle shape of the bulk solids has an influence on the acceleration behavior at the entry of pneumatic conveying systems as well as the particle/fluid behavior after bends, where the particles form ropes which disperse thereafter. The particle shape thereby directly effects the dispersion kinetics (see Figure 1a) and thereby the overall pressure drop, but also the stressing of the pipe walls which in consequence leads to possible erosion as well as attrition on the particle side. DEM-FVM simulations have advanced to the point that they can help optimize the transport of bulk solids in pneumatic conveying systems.

A second application are fluidized and packed beds (Figure 1b). Results for the pressure drop obtained experimentally for packings of both spherical and non-spherical particles match well with simulation results obtained by DEM-FVM (see Figures 1c and 1d). Currently for many particle shapes no correlations for pressure drop are available. Here DEM-FVM can contribute towards the prediction and optimization of pressure drop in much more complicated systems than shown in Figure $1 \mathrm{~b}$.

Particle resolved DEM-LBM based CFD is computationally much more demanding, however is not dependent on closures such as Eqs. (7)-(10). Static packing of particles (Figure 2a) can be investigated by particle resolved DEMLBM to obtain improved closures for momentum but also heat transfer (see e.g. (Kravets et al., 2019)). On the other hand, particle resolved DEM-LBM simulations allow a detailed insight into the dynamics of particle-fluid systems as all relevant interactions are resolved (Figure 2b). Such level of detail is required in several applications like e.g. micro fluidic devices.

a)

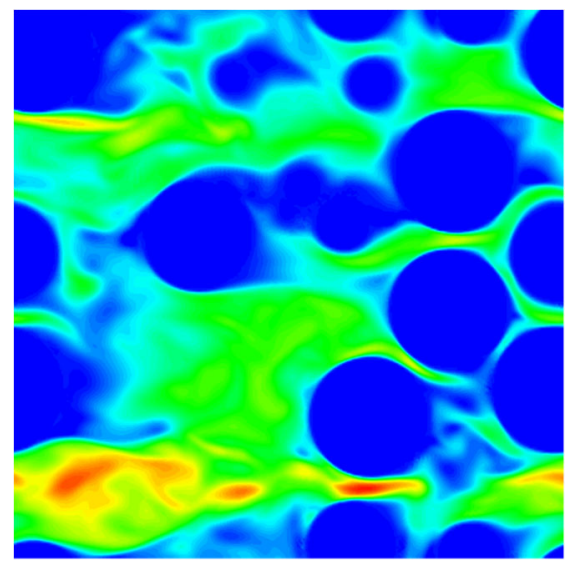

b)

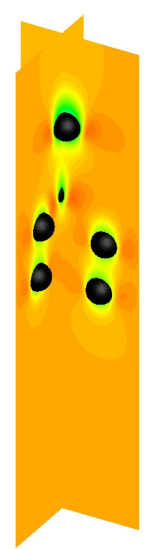

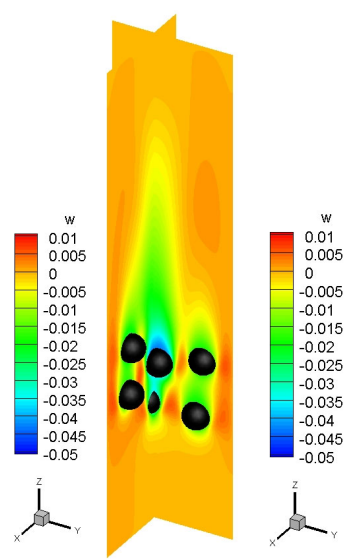

Figure 2. (a) Fluid velocities in a packing of spherical particles and (b) setup of moving particles modelled as part of the particle resolved DEM-LBM

\section{Conclusions}

A review of two state of the art methods to couple the DEM with computational fluid dynamics was made. Relevant aspects of the approaches were addressed in terms of the underlying framework of equations. Current applications were given. It is concluded that as computationally resources are still enhancing the number of possible applications of the discussed approaches is expected to grow significantly in the future making their improvement a relevant emerging field.

\section{Acknowledgements}

Financial support by DFG projects KR3446/6-1, KR3446/13-1 and KR3446/14-1 is gratefully acknowledged.

\section{References}

Cleary, P. W. (2008). The effect of particle shape on simple shear flows. Powder Technology, 179(3), 144-163. https://doi.org/10.1016/j.powtec.2007.06.018

Cundall, P. A., \& Strack, O. D. L. (1979). A discrete numerical model for granular assemblies. Géotechnique, 29(1), 47-65. https://doi.org/10.1680/geot.1979.29.1.47

d'Humières, D. (2002). Multiple-relaxation-time lattice Boltzmann models in three dimensions. Philosophical Transactions of the Royal Society of London. Series A: Mathematical, Physical and Engineering Sciences (Eds. P. V. Coveney et al.), 360(1792), 437-451. https://doi.org/10.1098/rsta.2001.0955

Di Felice, R. (1994). The voidage function for fluid-particle interaction systems. International Journal of Multiphase Flow, 20(1), 153-159. https://doi.org/10.1016/0301-9322(94)90011-6 
He, Y.-L., Liu, Q., Li, Q., \& Tao, W.-Q. (2019). Lattice Boltzmann methods for single-phase and solid-liquid phase-change heat transfer in porous media: A review. International Journal of Heat and Mass Transfer, 129, 160-197. https://doi.org/10.1016/j.jiheatmasstransfer.2018.08.135

Hilton, J. E., Mason, L. R., \& Cleary, P. W. (2010). Dynamics of gas-solid fluidised beds with non-spherical particle geometry. Chemical Engineering Science, 65(5), 1584-1596. https://doi.org/10.1016/j.ces.2009.10.028

Höhner, D., Wirtz, S., Kruggel-Emden, H., \& Scherer, V. (2011). Comparison of the multi-sphere and polyhedral approach to simulate non-spherical particles within the discrete element method: Influence on temporal force evolution for multiple contacts. Powder Technology, 208(3), 643-656. https://doi.org/10.1016/j.powtec.2011.01.003

Hölzer, A., \& Sommerfeld, M. (2008). New simple correlation formula for the drag coefficient of non-spherical particles. Powder Technology, 184(3), 361-365. https://doi.org/10.1016/j.powtec.2007.08.021

Kravets, B., Rosemann, T., Reinecke, S. R., \& Kruggel-Emden, H. (2019). A new drag force and heat transfer correlation derived from direct numerical LBM-simulations of flown through particle packings. Powder Technology, 345, 438-456. https://doi.org/10.1016/j.powtec.2019.01.028

Kruggel-Emden, H., \& Oschmann, T. (2014). Numerical study of rope formation and dispersion of non-spherical particles during pneumatic conveying in a pipe bend. Powder Technology, 268, 219-236. https://doi.org/10.1016/j.powtec.2014.08.033

Kruggel-Emden, H., Simsek, E., Rickelt, S., Wirtz, S., \& Scherer, V. (2007a). Review and extension of normal force models for the Discrete Element Method. Powder Technology, 171(3), 157-173. https://doi.org/10.1016/j.powtec.2006.10.004

Kruggel-Emden, H., Wirtz, S., \& Scherer, V. (2007b). An analytical solution of different configurations of the linear viscoelastic normal and frictional-elastic tangential contact model. Chemical Engineering Science, 62(23), 6914-6926. https://doi.org/10.1016/j.ces.2007.08.049

Markauskas, D., Kačianauskas, R., Džiugys, A., \& Navakas, R. (2010). Investigation of adequacy of multi-sphere approximation of elliptical particles for DEM simulations. Granular Matter, 12(1), 107-123. https://doi.org/10.1007/s10035-009-0158-y

Oschmann, T., Hold, J., \& Kruggel-Emden, H. (2014). Numerical investigation of mixing and orientation of non-spherical particles in a model type fluidized bed. Powder Technology, 258, 304-323. https://doi.org/10.1016/j.powtec.2014.03.046

Peng, Ch., Teng, Y., Hwang, B., Guo, Z., \& Wang, L.-P. (2016). Implementation issues and benchmarking of lattice Boltzmann method for moving rigid particle simulations in a viscous flow. Computers and Mathematics with Applications, 72(2), 349374. https://doi.org/10.1016/j.camwa.2015.08.027

Ren, B., Zhong, W., Chen, Y., Chen, X., Jin, B., Yuan, Z., \& Lu, Y. (2012). CFD-DEM simulation of spouting of corn-shaped particles. Particuology, 10(5), 562-572. https://doi.org/10.1016/j.partic.2012.03.011

Succi, S. (2008). Lattice Boltzmann across scales: From turbulence to DNA translocation. European Physical Journal B, 64(3-4), 471-479. https://doi.org/10.1140/epjb/e2008-00067-3

Succi, S. (2015). Lattice Boltzmann 2038. EPL (Europhysics Letters), 109(5), 50001. https://doi.org/10.1209/0295-5075/109/50001

Tao, S., Hu, J., \& Guo, Z. (2016). An investigation on momentum exchange methods and refilling algorithms for lattice Boltzmann simulation of particulate flows. Computers and Fluids, 133, 1-14. https://doi.org/10.1016/j.compfluid.2016.04.009

Verhaeghe, F., Luo, L. S., \& Blanpain, B. (2009). Lattice Boltzmann modeling of microchannel flow in slip flow regime. Journal of Computational Physics, 228(1), 147-157. https://doi.org/10.1016/j.jcp.2008.09.004

Wen, B., Zhang, Ch., Tu, Y., Wang, Ch., \& Fang, H. (2014). Galilean invariant fluid-solid interfacial dynamics in lattice Boltzmann simulations. Journal of Computational Physics, 266, 161-170. https://doi.org/10.1016/j.jcp.2014.02.018

Zhou, Z. Y., Pinson, D., Zou, R. P., \& Yu, A. B. (2011). Discrete particle simulation of gas fluidization of ellipsoidal particles. Chemical Engineering Science, 66(23), 6128-6145. https://doi.org/10.1016/j.ces.2011.08.041

Zhu, H. P., Zhou, Z. Y., Yang, R. Y., \& Yu, A. B. (2007). Discrete particle simulation of particulate systems: Theoretical developments. Chemical Engineering Science, 62(13), 3378-3396. https://doi.org/10.1016/j.ces.2006.12.089 